\title{
CONF-950846--70
}

UCRL-JC-121636

PREPRINT

\section{High Energy-Density Physics: From Nuclear Testing to the Superlasers}

E. Teller, E. M. Campbell, N. C. Holmes, S. B. Libby, and B. A. Remington
RECEIVED

NOV 171995

OSATI

This paper was prepared for submittal to the

1995 APS Topical Conference

Seattle, Washington

August 13-18, 1995

August 14, 1995

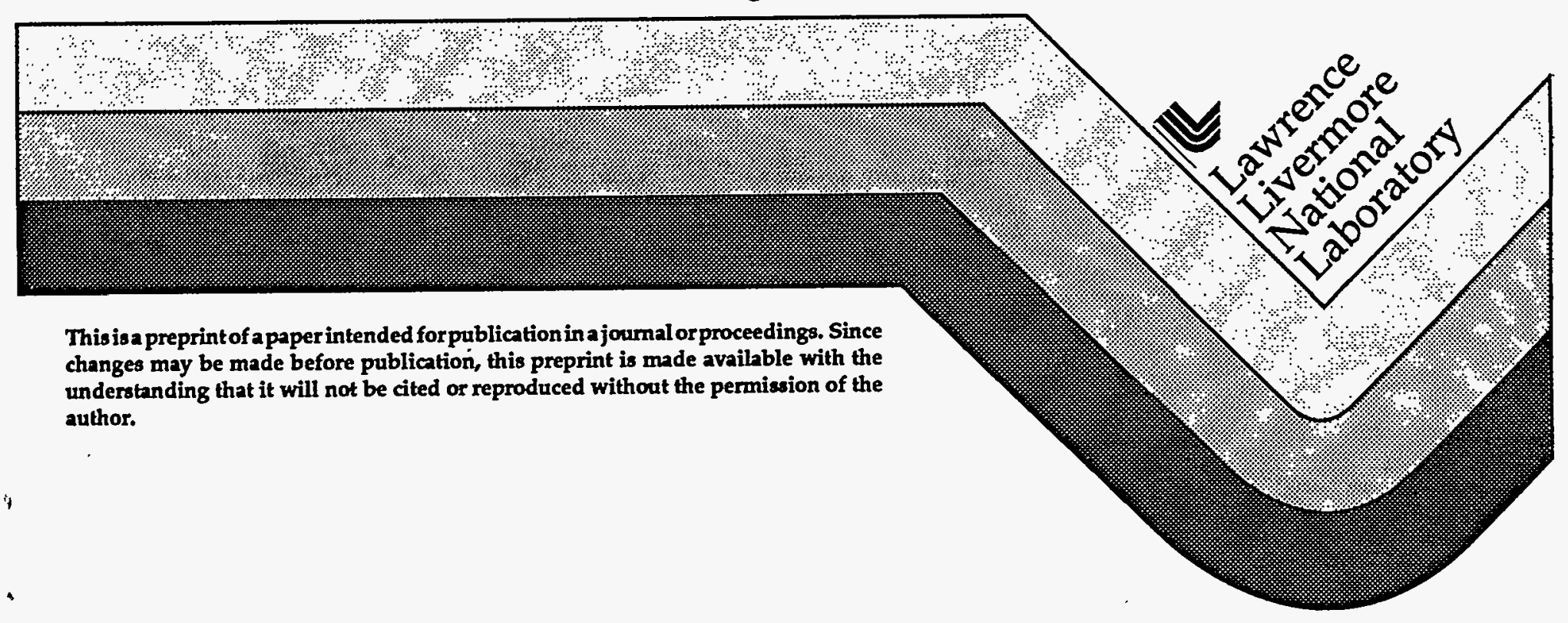




\section{DISCLAIMER}

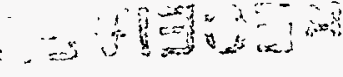

This document was prepared as an account of work sponsored by an agency of the United States Government. Neither the United States Government nor the ज政 1 के University of California nor any of their employees, makes any warranty, express or implied, or assumes any legal liability or responsibility for the accuracy, completeness, or usefulness of any information, apparatus, product, or process now disclosed, or represents that its use would not infringe privately owned rights. Reference herein to any specific commercial product, process, or service by trade name, trademark, manufacturer, or otherwise, does not necessarily constitute or imply its endorsement, recommendation, or favoring by the United States Government or the University of California. The views and opinions of authors expressed herein do not necessarily state or reflect those of the United States Government or the University of California, and shall not be used for advertising or product endorsement purposes. 


\section{DISCLAMMER}

Portions of this document may be illegible in electronic image products. Images are produced from the best available original document. 


\title{
HIGH ENERGY-DENSITY PHYSICS: FROM NUCLEAR TESTING TO THE SUPERLASERS
}

\author{
E. Teller, E. M. Campbell, N. C. Holmes, S. B. Libby, and B. A. Remington
}

Lawrence Livermore National Laboratory, Livermore, CA 94550

\begin{abstract}
We describe the role for the next-generation "superlasers" in the study of matter under extremely high energy density conditions, in comparison to previous uses of nuclear explosives for this purpose. As examples, we focus on three important areas of physics that have unresolved issues which must be addressed by experiment: equations of state, turbulent hydrodynamics, and the transport of radiation. We will describe the advantages the large lasers will have in a comprehensive experimental program.
\end{abstract}

\section{INTRODUCTION}

It has become apparent that in the event of a comprehensive nuclear test ban treaty, mankind would lose access to a regime of high energy-density physics that has been difficult to attain by other known means. This has led to a proposal from Chelyabinsk, Russia to resume nuclear testing, but purely for scientific research purposes.(1) However, with the advent of the proposed new generation "superlasers" such as the U.S. National Ignition Facility (NIF),(2) and the French Laser Megajoule Project(3) (LMJ), the capability to focus 1-2 megajoules of energy into sub-millimeter-scale volumes at power levels of $500 \mathrm{TW}$ will become routine. These lasers are being built to spearhead the international effort in controlled nuclear fusion through inertial confinement fusion(4,5) (ICF) and, indeed, to open new regimes for high energy density physics research.(6) With the advent of megajoule class lasers, one recovers the ability to access very high energy-density regimes hitherto extant only at the cores of stars and in nuclear detonations. The question to be briefly explored in this paper is exactly what are some of the regimes that the superlasers can access that were previously achievable only in a nuclear experiment. In a brief discourse, this topic obviously cannot be treated in a comprehensive fashion. Instead, we will mention only three areas as examples: equation of state (EOS), turbulent hydrodynamics, and radiation physics. These topics are representative of areas that have already been studied at Nova class lasers.(6) A wide range of other possible topics; suchs as plasma physics with $10^{7}-$ $10^{8}$ gauss local magnetic fields, or experiments with the intense $\left(10^{18}\right)$ neutron burst expected from the NIF capsule ignition will not be addressed here. We will close with a brief discussion of some of the advantages of the superlasers.

\section{EQUATION OF STATE}

One of the main goals of any high pressure research program is to understand the compressibility of matter under high dynamic loading using shock waves or isentropic compression. Among the many applications include: basic condensed matter physics, planetary physics, geophysics, astrophysics, and laboratory-scale fusion. Other high-energy density research problems, such as radiation transport and hydrodynamics, are sensitive to the state of the materials under study. While there is no doubt that the nuclear approach is highly developed and has been very successful, we believe that, in many cases, an equally strong program can be developed without the use of nuclear devices. The scale of NIF and other "superlasers," in terms of sample size and time scale, 


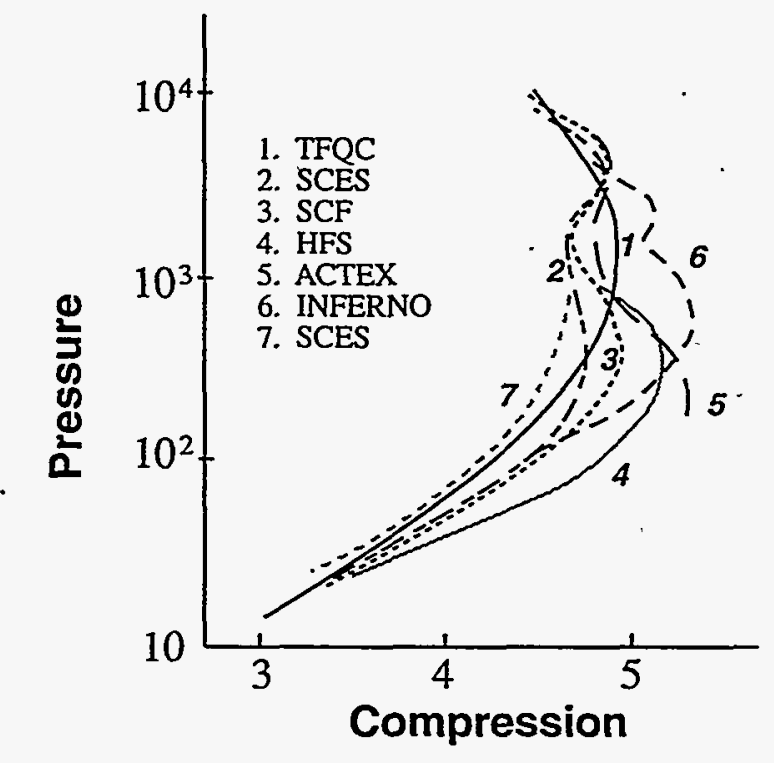

FIGURE 1. Calculations of the principal Hugoniot of aluminum using a variety of theoretical methods. The pressure is expressed in Mbar. TFQC is a Thomas-Fermi model, numbers 2, 3, 7 are selfconsistent field models, HFS is a Hanree-Fock-Slater model, Actex is an underdense plasma theory (basically an opacity model), INFERNO is an atom-in jellium model. Note that the uncertainty in density in the range of a few hundred Mbar is roughly $25 \%$. This result is typical of most metals in this pressure range. This figure is reproduced from Ref. 9.

considered together with the highly developed diagnostic tools developed by laser-fusion efforts worldwide, make credible a laboratory-scale effort.

Russian scientists have measured the equation of state of aluminum at pressures as high as $400 \mathrm{TPa}(4$ Gbar).(7)Recent laser experiments have achieved comparable pressures of $75 \mathrm{TPa}$ in gold.(8) This is a range of pressures for which both temperature and pressure ionization effects dominate the equation of state. However, no experiments have yet proven accurate enough to discriminate between the many competing theoretical treatments, shown schematically in Fig. 1.(9) Further development is needed.

The main difficulty in EOS experiments is determining the Hugoniot absolutely by simultaneous measurements of mass and shock velocity. This has been done successfully in nuclear driven experiments using gamma-reference layers( $(9)$ or neutron resonance Doppler shifts.(10) In the case of lasers, the method of side-lighting a moving layer by $\mathrm{x}$-rays is well established; it must be brought to a high level of accu- racy. It is important to note that for many metals at these pressures, the temperature is even more uncertain than the density, which leads us to consider possible simultaneous opacity experiments to characterize the temperature. An alternative approach for making high pressure EOS measurements is to use satellite-borne collision experiments in near-Earth or other orbits to enable collisions at extremely high relative velocities.(11) This may serve equally well at comparable cost to nuclear experiments, and may achieve significantly better absolute accuracy. Note also that at extremely high pressures, radiative preheat of the material ahead of the shock front could become a significant effect.(12) .

We often want to test materials off the principal Hugoniot, for example, on the isentropic release from shocked states, or on isochoric paths from normal densities. This can be performed in a variety of ways using the NIF, whose scale will make accurate measurements possible.(6) For example, shock and release into low-density foams,(13) or shock compression of highly porous materials(14) offer complementary approaches toward achieving the desired states of matter. Most planetary or astrophysicall applications lie on isentropic compression paths.(15) This is an area which has been studied mostly with static compression in diamond-anvil cells, which access pressures up to $\sim 0.3 \mathrm{TPa}$.(16) Above this pressure, laser experiments will be most useful. This is an area we want to emphasize as a frontier of high pressure physics. And this is the area in which the NIF should excel, since most of the development has been aimed at such states. Large lasers have been driven by the requirements of the laser fusion program, and their application to other areas of fundamental research has yet to be explored fully. For example, electrical and thermal conductivities are unknown in this region. Magnetic properties are unknown, although maintaining sufficiently low temperatures will be a great challenge. Likewise, we simply don't know the details of material structure at these conditions. It is likely that many new and exciting phase transitions may be found, and not only as a result of pressure, but also in high magnetic fields, for example.

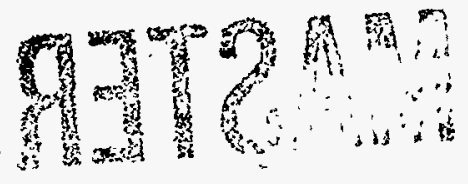




\section{TURBULENT HYDRODYNAMICS}

From situations as commonplace as the turbulent mixing of gasoline with air in the carburetor of your car, to the violent core-envelope mixing that leads to the cataclysmic stellar explosions of superno.vas,(17) one is surrounded with examples of non-linear hydrodynamic mixing. However, the area of turbulent hydrodynamics and material mixing remains one of the most theoretically intractable problems. Experimental facilities in relevant regimes are essential to benchmark various modeling techniques.

One long-used method of investigating shockinduced compressible mixing is the use of shock tubes. Here, the acceleration is impulsive, the pressures are typically only a few bar, the compression is low, the amount of perturbation growth is modest (growth factor of a few), and there is no radiation or ionization. High explosives generate pressures up to 200-300 $\mathrm{kbar}$, but compression is low, there is no ionization or radiation, and diagnostic access is limited. Gas guns can generate pressures up to $1 \mathrm{Mbar}$, large perturbation growth, but with modest compression, diagnosis is difficult, and there is no radiation or ionization. Macroscopic cell accelerators deal with incompressible hydrodynamics, modest accelerations (10$1000 \mathrm{~g}_{\mathrm{o}}$ ), growth factors can be large, diagnosis is good, but there is no radiation or ionization. On large lasers like Nova and the NIF, the accelerations are extreme $\left(10^{13}-10^{14} \mathrm{~g}_{\mathrm{o}}\right)$, pressures of 100 's of Mbar can be achieved, and one has high growth factors, very large compression, and high levels of radiation and ionization. The situation in a nuclear detonation is similar, only all of the scales are larger. The issue of macroscopic (say, as in nuclear testing) vs. microscopic (such as Nova or NIF) experiments needs to be addressed. As an example, we show in Fig. 2a typical data from a macroscopic $(12 \mathrm{~cm})$ water cell experiment(18) and in Fig. 2b a microscopic $(500 \mu \mathrm{m})$ Nova experiment,(19) both looking at the 3D Rayleigh-Taylor evolution of a square $k_{x}=k_{y}$ surface perturbation. The water experiment was done at an acceleration of $10 \mathrm{~g}_{0}$ with no compression, no radiation, and was diagnosed by side-on optical shadowgraphy. The Nova experiment was done at $10^{13} \mathrm{~g}_{\mathrm{o}}$ acceleration under high compression (5-10

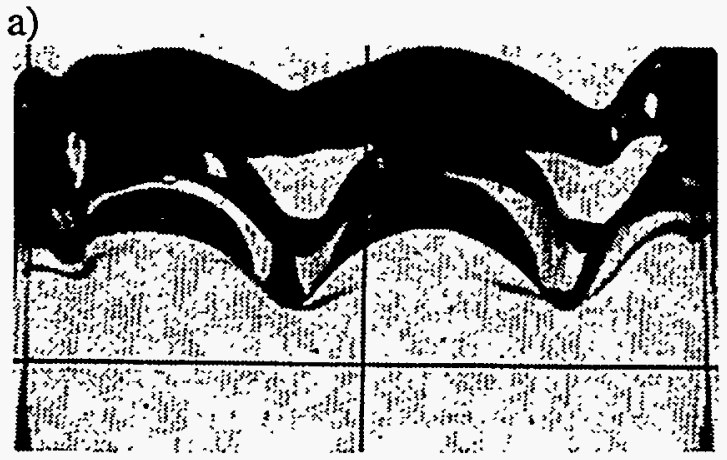

b)

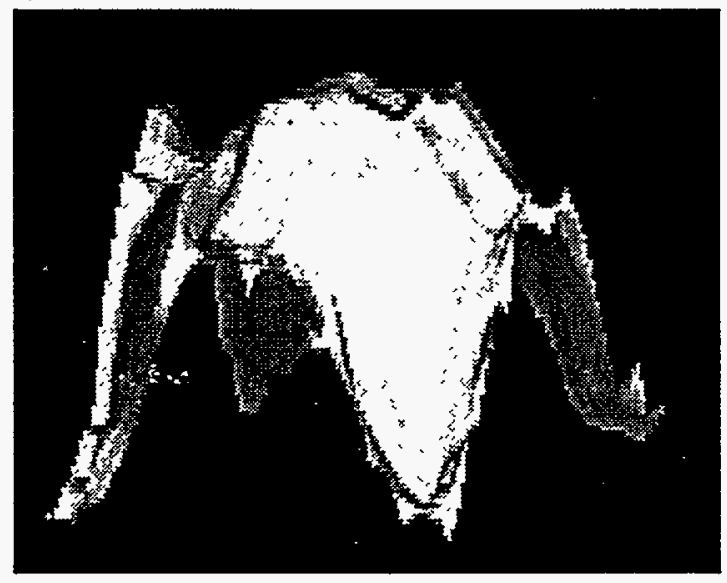

FIGURE 2. (a) Comparison of 3D single-mode RayleighTaylor data taken on a $12 \mathrm{~cm}, 10 \mathrm{~g}_{\mathrm{a}}$ macroscopic water-cell accelerator (top figure); (b) the lower figure data from a NOVA experiment.

times solid), with high levels of radiation flow, and diagnosed by face-on $\mathrm{x}$-ray back lighting. As one can see from comparing Figs. $2 a$ with $2 b$, the microscopic imaging capabilities on Nova are indeed impressive,(20) with as much detail observable in the Nova experiment as in the macroscopic water experiment. Therefore, the microscopic nature of Nova and NIF experiments does not present an insurmountable hindrance to the pursuit of fundamental questions of interest to high energy-density physics. 


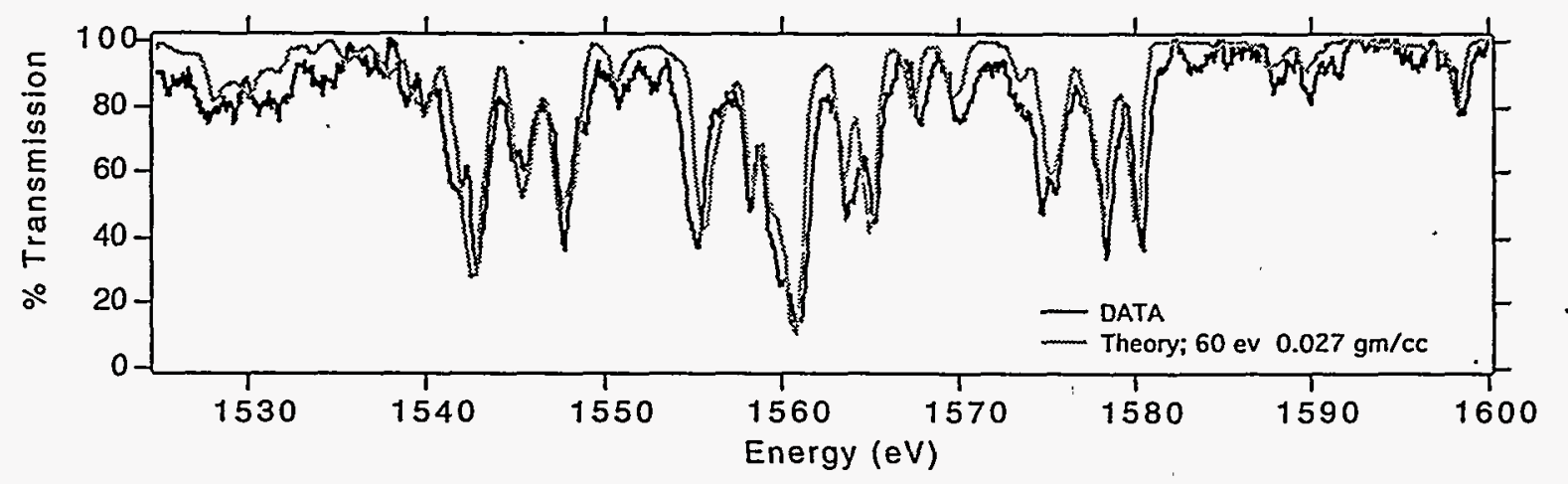

FIGURE 3. Nova experimental data for the LTE opacity of aluminum compared to the OPAL code.

\section{RADIATION PHYSICS}

The study of the radiation physics is a central part of high energy density research. Almost by definition, "high energy density physics," denotes a regime where the emission and absorption of radiation (usually $\mathrm{x}$-rays) from stripped ions, and the transport of that radiation forms an important part of the energy balance of the medium.

The study of radiation physics can be described in three somewhat interwoven categories. The first is the study of the radiative properties of stripped atoms in plasmas, which ranges from the study of the atomic spectroscopy of isolated ions, to the study of complex radiation opacities. The latter involves consideration of an enormous number of relevant ionic states and transitions (up to $10^{8}$ in the case of M-band dominated opacities) and the effects of plasma on them (21). The second category involves the application of such radiative properties to situations such as the behavior of an ICF hohlraum; or the role of metal opacities in stellar models such as those for Cepheid variable.pulsation (22). Finally, the third category is the practical application of radiation physics to the development of new techniques for plasma diagnostics and potentially other fields such as medical physics (23).

As an example of the "second category," experiments done at the Nova laser have already demonstrated (24) the ability to prepare a uniform, $x$-ray heated sample in local thermodynamic equilibrium (LTE), and to measure its opacity through a point projection spectroscopic method. Figure 3 shows the results for such a transmission spectrum for aluminum at about $60 \mathrm{eV}$ compared to calculations using the detailed accounting code OPAL (22). This method was also used to verify the OPAL predictions for $\mathrm{Fe}$ opacities at astrophysically interesting $\mathrm{x}$-ray energies (24). It is worth mentioning that the code result and its experimental verification turned out to be so precise that the results can be used as a thermometer that is accurate to a few percent for use in other experiments

An example from our "third category" of the feedback of laser physics experiments into a useful diagnostic lies in the non-LTE plasma experiments that led to the discovery and development of $x$-ray lasers at Nova and other large lasers (25). In figure 4 we

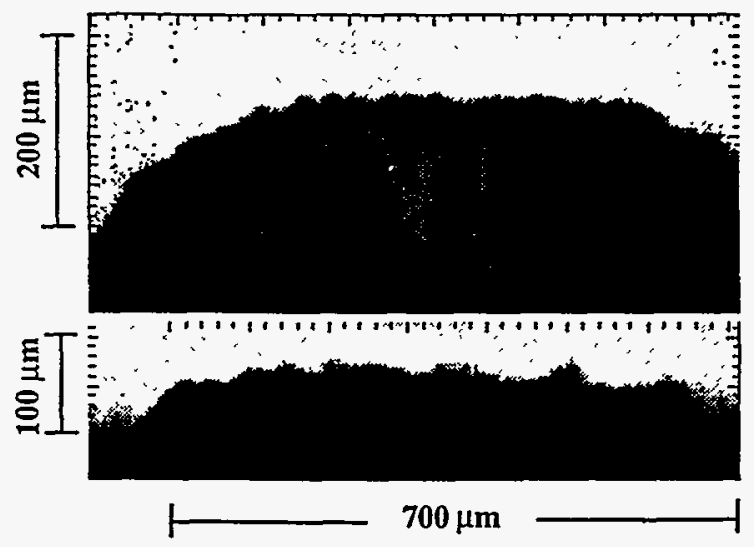

FIGURE 4. Fine structure in the plasma blowoff on the rear side of a foil irradiated with a $1 \mathrm{~ns} 2 \omega$ laser pulse at intensity of $10^{14} \mathrm{~W} /$ $\mathrm{cm}^{2}$. The magnification was $30 \mathrm{x}$, allowing $5 \mu \mathrm{m}$ features to be resolved. 
show how the x-ray laser's extraordinary effective brightness ( $\sim \mathrm{GeV} / \mathrm{cm}^{2}$-sec-ster) is being used to $\mathrm{im}$ age the column densities of hot dense plasmas down to the micron scale with 50 ps time resolution (26). From these examples of work done at Nova (operating from $~ 1$ to 50 kilojoules) and other lasers, we may infer that the megajoule class lasers will allow us to extend the study of radiative properties of LTE and non-LTE matter to far higher $Z$, densities, and temperatures, as well as to use these new radiative sources as diagnostics.

\section{SUMMARY}

Nuclear experiments offer the unique possibility of bringing very large volumes of material into high energy density conditions, and experiments that require that feature are likely to remain solely in the province of nuclear experimentation. However, such experiments are expensive, and are difficult to diagnose with high precision. In contrast, experiments with the superlasers such as the NIF can conduct experimental campaigns at a shot rate of over 4 shots per day. Therefore, the laser experiments offer the possibility of extensive parameter variation, control, and diagnostic development. Examples of this ability to control the experiments are: the ubiquitous use of timed back lighters to "photograph" the hydrodynamic instability of the sort shown in figure 2 , or to measure the transmission spectrum of the LTE opacity sample shown in figure 3 . Another, less obvious advantage of the laser based experiments is the ease of preparing samples that are optically thin and are thus fully diagnosable. For example, analysis of hydrodynamic instabilities such as that shown in figure 2 require in general a three dimensional reconstruction of the target. This is only possible for samples that are optically thin to the relevant backlighter.

\section{ACKNOWLEDGMENTS}

This work was performed under the auspices of the U.S. Department of Energy by the Lawrence Livermore National Laboratory under contract No. W-7405-ENG-48.

\section{REFERENCES}

1. E. N. Avrorin, B.V. Litvinov, and V.A. Simonenko, proposal,
"Uses of Nuclear Explosivions for Fundamental Research" (1995), in preparation.

2. J.A. Paisner, E.M. Campbell, and W.J. Hogan, Fusion Technology 26, 755 (1994).

3. CHOCS No. 13, Avril 1995. Revue Scientifique et Technique de la Direction des Applications Militaires, CEA; M. Andre, "ConceptualDesign of the French LMJ Laser," Ist Annual Intemational Conference on Solid State Lasers for Application to ICF, Montcrey, CA, May 31 -June I (1995). SPIE proceedings.

4. J. Nuckolls et al., Nature 239, 139 (1972).

5. J. D. Lindl and W. C. Mead, Phys. Rev. Lett. 34, 1273 (1975).

6. R. W. Lee et. al., "Science on High Energy Lasers From Today to the NIF," UCRL-ID-119170.

7. V. A. Simonenko, N. P. Voloshin, A. S. Valdimirov, A.P. Nagibin, V. N. Nogin, V. A. Popov, V. A. Vasilenko, and Yu. A. Sholdin, Sov. Phys. JETP 61,869(1985).

8. R. W. Cauble, D. W. Phillion, T. J. Hoover, N. C. Holmes, J. D. Kilkenny, and R. W. Lec, Physs. Rev. Lett. 70, 2102 (1993).

9. E. Avrorin, B. K. Vodologa. N. P. Voloshin, V.. F. Kuropatenko, G. V. Kovalenko, V. A. Simonenko, and B. T. Chemovolyuk, Sov: Phrsics JETP 66, 348 (1987).

10. C. E. Ragan III, M. G. Silbert. and B. C. Diven, J. Appl. Phys. 48, 2860(1977).

11. R. M. More. N. C. Holmes, and R. Hyde, unpublished.

12. Ya. B. Zel'dovich and Yu. P. Raizer, Physics of Shock Waves and High-Temperature Hydrodynamic Phenomena, Academic Press (New York) 1967

13. N.C. Holmes. H.B. Radousky, M.J. Moss and W.J. Nellis, Appl. Phws. Lent 45, 626(1984); N. C. Holmes, in High-Pressure Sceince and Teclmology-1993, Schmidt, Shaner, Samara, Ross, eds. American Institute of Physics. New York, 1991, p. 153

14. Ya. B. Zel'dovich, Sol: Phys. JETP 5,1103 (1957).; N.C. Holmes, Rev. Sci. Instrum. 62,1990(1991); I. V. Lomonosov, A. V. Bushman. and V. E. Fortov, in High-Pressure Sceince and Techology-1993, Schmidt, Shaner, Samara, Ross, eds. American Institute of Physics. New York, 1991, p. 117-124.

15. W. B. Hubbard and R. Smoluchowski, Space Science Reviews 14, 599 (1973): D. J. Stevenson, Annual Reviews of Earth and Planetan: Sciences 10, 257 (1982).

16. J. Akella. High Pressure Science and Technology - 1993, ed. by S. C. Schmidt et al. (AIP, New York, 1993), p. 187.

17. E. Muller. B. Fryxell, and D. Amelt, Astron. Astrophys. 251 , 505 (1991).

18. Jacobs and Catton. J. Fluid Mech. 187, 353 (1988).

19. Marinak et al., submitced to Phys. Rev. Lett. (1995); B.A. Remington et al.. Phys. Plasmas 2, 241 (1995).

20. J.D. Kilkenny. Rev. Sci. Instrum. 63, 4688 (1992).

21. A. Bar-Shalom, J. Oreg, W. H. Goldstein, D. Shvarts, and A. Zigler, Phys. Rev. A. 40.3183(1989).

22. F. J. Rogers and C. A. Iglesias, Science 263,50(1994).

23. L. B. DaSilva et al., Science 258, 269 (1992).

24. T. S. Perry et. al, " Plyss. Rev. Let., 67, 3784, (1991); L. Da Silva et. al.. Phys. Rev. Lett. 69, 438, (1992); P. T. Springer et. al. Phys. Rev: Lell., 69, 3735, (1992).

25. R. Elion. $X$-Ray Lasers, New York, Academic Press, 1990; D. C. Eder and D. L. Mathews, X-ray Lasers 1994 - 4th International Colloguium, (AIP Conference Proceedings 332, 1994).

26. R. C. Cauble et al., Phys. Rev. Lett. 74, 3816 (1995). 